\title{
Regional Differences in the Sensitivity of MEG for Interictal Spikes in Epilepsy
}

\author{
Geertjan Huiskamp • Zaloa Agirre-Arrizubieta • \\ Frans Leijten
}

Received: 29 August 2009/Accepted: 1 February 2010/Published online: 12 February 2010

(C) The Author(s) 2010. This article is published with open access at Springerlink.com

\begin{abstract}
MEG interictal spikes as recorded in epilepsy patients are a reflection of intracranial interictal activity. This study investigates the relationship between the estimated sources of MEG spikes and the location, distribution and size of interictal spikes in the invasive ECoG of a group of 38 epilepsy patients that are monitored for presurgical evaluation. An amplitude/surface area measure is defined to quantify and rank ECoG spikes. It is found that all MEG spikes are associated with an ECoG spike that is among the three highest ranked in a patient. Among the different brain regions considered, the fronto-orbital, interhemispheric, tempero-lateral and central regions stand out. In an accompanying simulation study it is shown that for hypothesized extended sources of larger sizes, as suggested by the data, source location, orientation and curvature can partly explain the observed sensitivity of MEG for interictal spikes.
\end{abstract}

Keywords MEG $\cdot$ ECoG $\cdot$ Epilepsy $\cdot$ Interictal . Distributed source

\section{Introduction}

Interictal spikes are hallmarks of epilepsy in the EEG and MEG that are measured in a patient in the period between

This is one of several papers published together in Brain Topography on the "Special Topic: Cortical Network Analysis with EEG/MEG'.

G. Huiskamp $(\bowtie) \cdot$ Z. Agirre-Arrizubieta $\cdot$ F. Leijten Department of Neurology and Clinical Neurophysiology, Rudolf Magnus Institute of Neuroscience, University Medical Center Utrecht, F02.230 Heidelberglaan 100, 3584 CX Utrecht,

The Netherlands

e-mail: ghuiskam@umcutrecht.nl seizures. The field distribution of these spikes shows a dipolar distribution, suggesting a focal origin, but as opposed to evoked- or event related signals these spontaneous signals can have a relatively large amplitude $(\sim 100$ $\mu \mathrm{V}$ in EEG, $\sim 3 \mathrm{pT}$ in MEG). This suggests that the generators of these signals must have a relatively large extent.

In some patients that do not respond to pharmacological treatment epilepsy surgery is an option. In the pre-surgical evaluation of such patients sometimes electrodes are implanted on the cortical surface for an extended period (5-10 days) in order to investigate which area in the brain is generating these seizures. Interictal spikes recorded using this invasive technique (electro-corticography or $\mathrm{ECoG}$ ) can be considered the gold standard for evaluating the detection and localization capabilities of noninvasive EEG and MEG.

For such evaluation chronic simultaneous registrations would be ideal. For MEG these are difficult to obtain, and only for a limited time $(\sim 1 \mathrm{~h})$. For EEG this is feasible. Studies on simultaneous EEG and intracranial ECoG have suggested that interictal spikes detectable with EEG are generated by relatively large patches of at least $10 \mathrm{~cm}^{2}$ of activated cortex (Tao et al. 2005).

Since it has been shown that the yield of interictal spikes in EEG and MEG does not differ much (de Jongh et al. 2005), a similar relation between the area of activated cortex and spikes detected by MEG is suggested. However, since it is known that MEG is sensitive only to sources tangential to the cortical surface, i.e. sources in the sulci, the specific location and extend of such sources might be different as compared to EEG, which is mostly sensitive to sources on the gyri. Especially the influence of cancellation of sources of opposite orientation will be different.

In this study we investigated the relationship between interictal spikes as observed in MEG and those recorded 
separately during chronic ECoG acquisition in a large group of epilepsy patients (38). MEG recordings lasted at least $1 \mathrm{~h}$, and all interictal spikes present were marked, clustered, averaged and localized. We assume that during chronic ECoG registration all interictal spikes that can occur in each patient will be recorded. For each patient the different ECoG spike types were characterized by a measure capturing both spike amplitude and extent, and they were ranked according to this measure. MEG MUSIC localization was then compared to the ranked ECoG spikes and analyzed with respect to concordance per anatomical region (Agirre-Arrizubieta et al. 2009). Additionally, MEG simulations were performed for activated cortical patches of varying size and location in a realistic high resolution model of the cortical surface. Findings from the patient data were interpreted in terms of the modeling results, in particular by comparing cortical regions where MEG and ECoG concordance was high to regions where large patchsize based simulated MEG amplitudes were high.

\section{Materials and Methods}

\section{Patients}

38 patients were studied, all suffering from refractory focal epilepsy and considered candidates for chronic ECoG monitoring and subsequent surgery. Target areas for implantation included the frontal (36), temporal (32), central (21) parietal (22), occipital (3) and inter-hemispheric (18) regions, either in the left (23) or right (15) hemisphere. All patients were treated in the University Medical Centre in Utrecht, as part of the national Collaborative Dutch Epilepsy Surgery Program. The study was approved by the Medical Ethics Committee of the hospital. Informed consent for the study was obtained from all participants.

\section{MRI}

For each patient a whole-head high-resolution 3D T1 MRI sequence allowing accurate segmentation of the cortical grey matter was performed (resolution typically $1 \times 1 \times$ $1.5 \mathrm{~mm}$ ). For the simulation study the high quality 3DT1 ch2 (brain-only) set as available in the distribution of the MRICRON software was used (Rorden and Brett 2000). Segmentation and tessellation was performed using CURRYV3.0 software (Compumedics, Charlotte, NC).

\section{MEG}

A 151-channel whole-head axial gradiometer MEG (CTF, Port Coquitlam, BC) was recorded. Data sets of 10-15 min were recorded, until 60-90 min of MEG data had been obtained. Head position with respect to the MEG helmet was measured. Head-shape information was acquired for MRI co-registration.

Two clinical neurophysiologists independently reviewed interictal spikes in MEG datasets. Only interictal spikes from sets for which there was sufficient agreement (interobserver $\kappa>0.4$ ) were used for source modeling. Spatiotemporal clustering of consensus spikes was performed (van t' Ent et al. 2003) and spikes from each cluster were averaged.

The averaged clusters were modeled in CURRYV3.0 using a realistic skull model as extracted from MRI. The interval from the beginning of the rising slope of the spike to the spike maximum was used for a rotating dipoles MUSIC analysis (Mosher et al. 1992). Points on the cortical surface with an average distance of $3 \mathrm{~mm}$ were used for scanning. Locations for which the resulting MUSIC metric exceeded $2 / 3$ of the overall maximum were displayed on a cortical rendering from MRI.

\section{ECoG}

ECoG was performed with subdural grid electrodes (Ad-Tech, Racine, WI) with up to 80-120 electrodes per patient. Electrodes were embedded in silicone with a distance of $1 \mathrm{~cm}$ and a $3 \mathrm{~mm}$ contact surface. An extra-cranial reference on the contra-lateral mastoid was used. ECoG was continuously recorded $24 \mathrm{~h}$ a day for an average of 7 days. The electrodes were localized and visualized by comparing digital photographs during and after implantation and by a post-implantation CT scan that was matched to an MRI.

For each patient $1 \mathrm{~h}$ of representative samples including all the different interictal ECoG spikes observed during the recording period were collected. The different spike types were identified and averaged. For each averaged ECoG spike, the following combined amplitude and synchronous surface-area measure was calculated. First, for each patient, $2 \mathrm{~s}$ of representative background activity, not containing interictal spikes, was selected and its root mean square value (RMS_bg, all electrodes, all time samples) was computed. Then for each averaged spike an interval of $200 \mathrm{~ms}$ was chosen centered on the time of the maximum of the spike. The rms value (RMS_spike) for that interval was determined for each electrode. Also, for each electrode a baseline interval of $100 \mathrm{~ms}$ before the start of the spike was chosen and its rms value (RMS_base) was computed. Electrodes were marked as being involved in a spike if RMS_spike exceeded twice the overall RMS_bg but also twice the RMS_base for that electrode. For each spike the RMS_spike values were then integrated over the marked electrodes to form a single value (RMS_int). Spikes with at 
least one marked electrode were ranked from large to small RMS_int and used for subsequent association with MEG results. Thus the ranked RMS_int value reflects a reliable (with respect to background activity) estimate of both amplitude and extent of the spike.

\section{MEG-ECoG Association}

Specific anatomical regions, reflecting the practice of epilepsy surgery, were defined. The temporal lobe was divided into a lateral and a mesial region. The frontal lobe was divided into a fronto-orbital region and the inferior, medial and superior frontal gyri. The inter-hemispheric region ranged from the frontal to the occipital lobe. The central region consisted of the gyri lining the central sulcus. The parietal region consisted of the superior and inferior lobules and the parietal parts of the supra-marginal and angular gyri. The occipital region contained the convexity and posterior part of the occipital lobe.

For each patient each MEG MUSIC result and each ranked ECoG spike were allocated independently to these predefined anatomical regions. Then, the association between ECoG and MEG was made by checking if anatomical regions corresponded. Thus for the whole group of patients, the percentage of ECoG spikes that had a MEG associate, the ranking of these spikes and the regions in which there was correspondence were tabulated.

\section{MEG Simulation}

A high resolution triangular tessellation based on the high quality brain-only MRI was generated. The average node area was $6 \mathrm{~mm}^{2}$. A corresponding realistic inner skull surface was constructed and from this a boundary element model for forward computation of MEG was set up using CURRYV3.0. Realistic CTF MEG helmet gradiometer positions were defined. For this configuration a transfer function for a distributed dipole layer with assumed uniform strength of $200 \mathrm{pAm} / \mathrm{mm}^{2}$ (Hillebrand and Barnes
2002) defined on the cortical surface (left hemisphere only) was computed. In the uniform distributed dipole layer model the basic elements are not dipoles but true (triangular) patches for which the field strength is proportional to the solid angle of the patch with respect to an observation point (van Oosterom et al. 1995). It can be considered a proper model for a cortical layer with homogeneous density of pyramidal cells and it does not show the singular behavior that the dipole model shows with respect to the observation point. Centered around each triangular node on the cortical surface two larger patches extending to neighboring nodes of average area $\sim 300$ and $1,200 \mathrm{~mm}^{2}$, so $\sim 50$ and 200 elementary triangles, were set up. An example of a small and large patch around a node in the frontal medial gyrus is shown in blue in Fig. 1 in the left and right panel respectively. Note that the large patch extends over sulci with opposite orientation. For all patches the maximum absolute MEG field strength occurring in the gradiometers was computed. Resulting values were assigned to the center triangular node and plotted on a folded-out version of the cortical surface for visualization. Locations showing large and small values where compared to the correspondence of MEG MUSIC results and ECoG results in those same locations in the patient data.

\section{Results}

\section{MEG-ECoG Association}

In total 56 different MEG clusters were found in 33 patients. For 3 patients no reliable spike could be identified in MEG, in two other patients ECoG data was insufficient. When localized using MUSIC five clusters were found outside an area covered by grids, these were left out of the analysis. Each remaining cluster could be associated to at least one ECoG spike. Moreover, there was always an association with ECoG spikes having a high RMS_int value ranked 1st, 2 nd or 3 rd. There were no MEG results in
Fig. 1 In blue small (left panel) and large (right panel) patches of the uniform distributed dipole source as defined on the high resolution tessellation of the cortical surface depicted in red

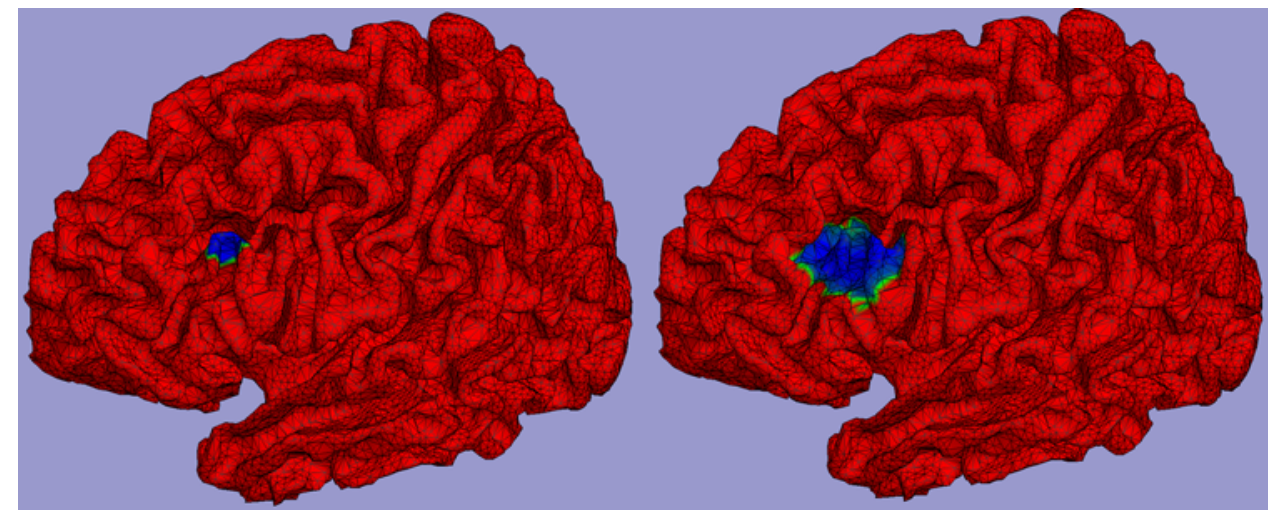


Fig. 2 Left panel MEG MUSIC result (purple) in the left lateral temporal lobe, for one cluster in one patient, displayed on the rendering of the cortical surface. Right panel $\mathrm{ECoG}$ result for the same patient, showing marked electrodes for three spikes ranked 1st (red), 2nd (green) and 3rd (blue), displayed on the matched CT-MRI rendering, in which the actual grid electrodes are visible
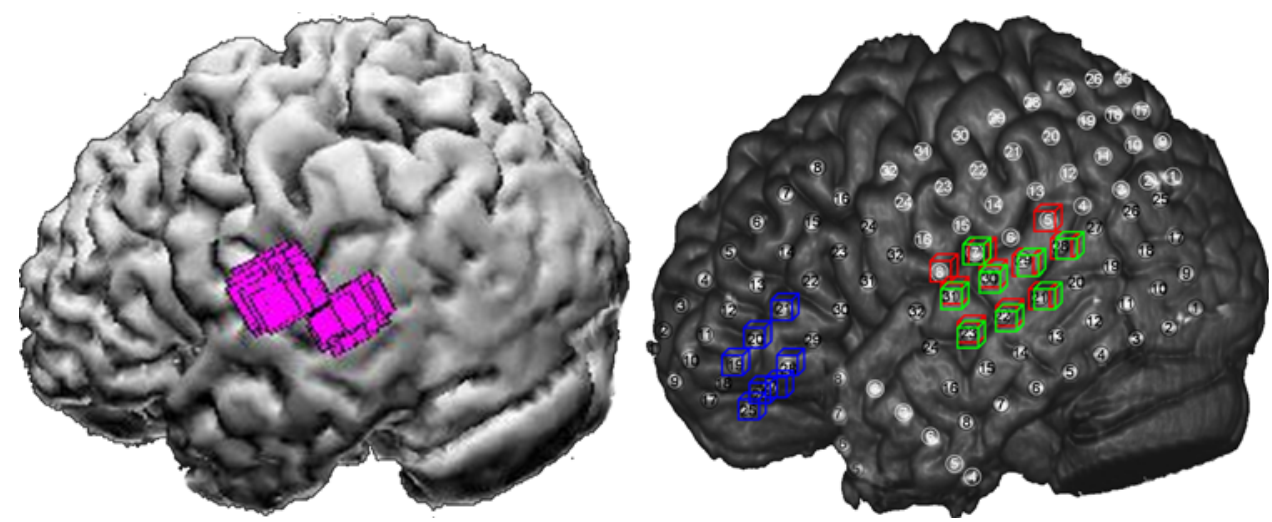

the area covered by grid-electrodes that did not have an ECoG associate. Conversely, of 221 ECoG spike types found 97 (44\%) did not have a MEG associate. An example of a MEG MUSIC result for one cluster in one patient is shown in Fig. 2 in the left panel. In the right panel three ECoG spikes ranked 1st (red), 2nd (green) or 3rd (blue) are shown. Note that here the MEG result in the left lateral temporal lobe can be associated to the red and green ECoG spike, but not to the blue one in the inferior frontal gyrus. The distribution of associations for the different regions is given in Table 1, and depicted in Fig. 3. Note that ECoG spikes can be attributed to more than one region. Only

Table 1 Numbers and percentages of EcoG spikes that were associated with a MEG cluster

\begin{tabular}{lllr}
\hline Anatomical region & $\begin{array}{l}\text { Total number } \\
\text { of ECoG spikes }\end{array}$ & $\begin{array}{l}\text { Number of } \\
\text { associated ECoG- } \\
\text { MEG spikes }\end{array}$ & $\%$ \\
\hline Fronto-orbital & 10 & 10 & 100 \\
Inter-hemispheric & 45 & 40 & 89 \\
Central & 42 & 32 & 76 \\
Temporal lateral & 79 & 58 & 73 \\
Frontal superior & 54 & 39 & 72 \\
Temporal mesial & 51 & 14 & 28 \\
\hline
\end{tabular}

Only areas where values were significant are given

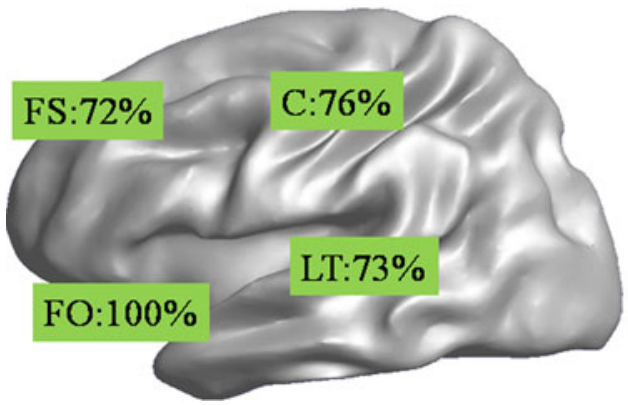

Fig. 3 Percentages of ECoG spikes that where associated with a MEG cluster for different regions, depicted on an unfolded cortex. Shown are a left lateral (left) and inter-hemispheric (right) view. significant values, with respect to the average value of $56 \%$ $(=100-44 \%)$, are given.

\section{MEG Simulation}

Maps of the maximum absolute MEG field strength for small $\left(\sim 300 \mathrm{~mm}^{2}\right)$ and large $\left(\sim 1200 \mathrm{~mm}^{2}\right)$ patches for all cortical points are shown in Fig. 4 in a left lateral and interhemispheric view. Maps are shown in a color coding scaled to the maximum value occurring. Maximum field strength for small patches was $370 \mathrm{fT}$, for large patches this was $950 \mathrm{fT}$. Note that for the large patches, maximum field strengths are larger, but only restricted to certain areas, whereas for small patches they are smaller but they are more evenly distributed, especially on the lateral cortex. For large patches, regions standing out are the occipital pole, followed by the temporal plane, the post-central sulcus, the fronto-orbital area, the temporal pole and the hemispheric region (superior frontal gyrus).

\section{Discussion}

Interictal spikes as detected in MEG are a reflection of interictal spikes as they occur in the ECoG: all MEG spikes that we found had an ECoG counterpart in the associated

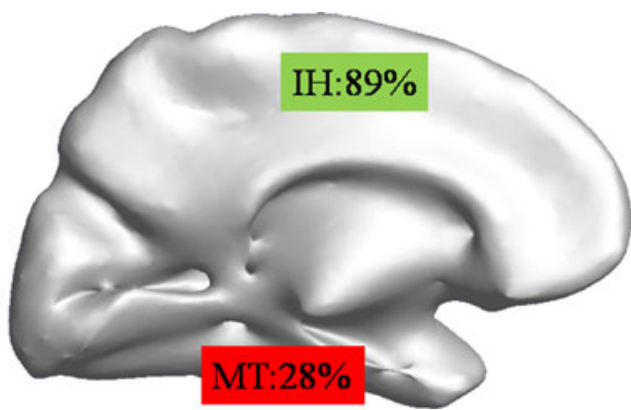

Regions indicated are the lateral temporal lobe (LT), central sulcus (C), fronto-orbital region (FO), inter-hemispheric region (IH), superior frontal gyrus (FS) and mesial-temporal region (MT) 
Fig. 4 Maximum MEG field strength for large $\left(\sim 1200 \mathrm{~mm}^{2}\right.$, upper panels) and small patches ( $\sim 300 \mathrm{~mm}^{2}$, lower panels). Shown are a left lateral (left) and inter-hemispheric (right) view. Color maps are scaled from zero (pink) to maximal value (blue) occurring (0.95 pT for large, $0.37 \mathrm{pT}$ for small patches). Increased sensitivity for large patches exists in the occipital pole (I), temporal plane (II), post-central sulcus (III), fronto-orbital (IV), temporal pole $(\mathrm{V})$ and interhemispheric region/superior frontal gyrus (VI)
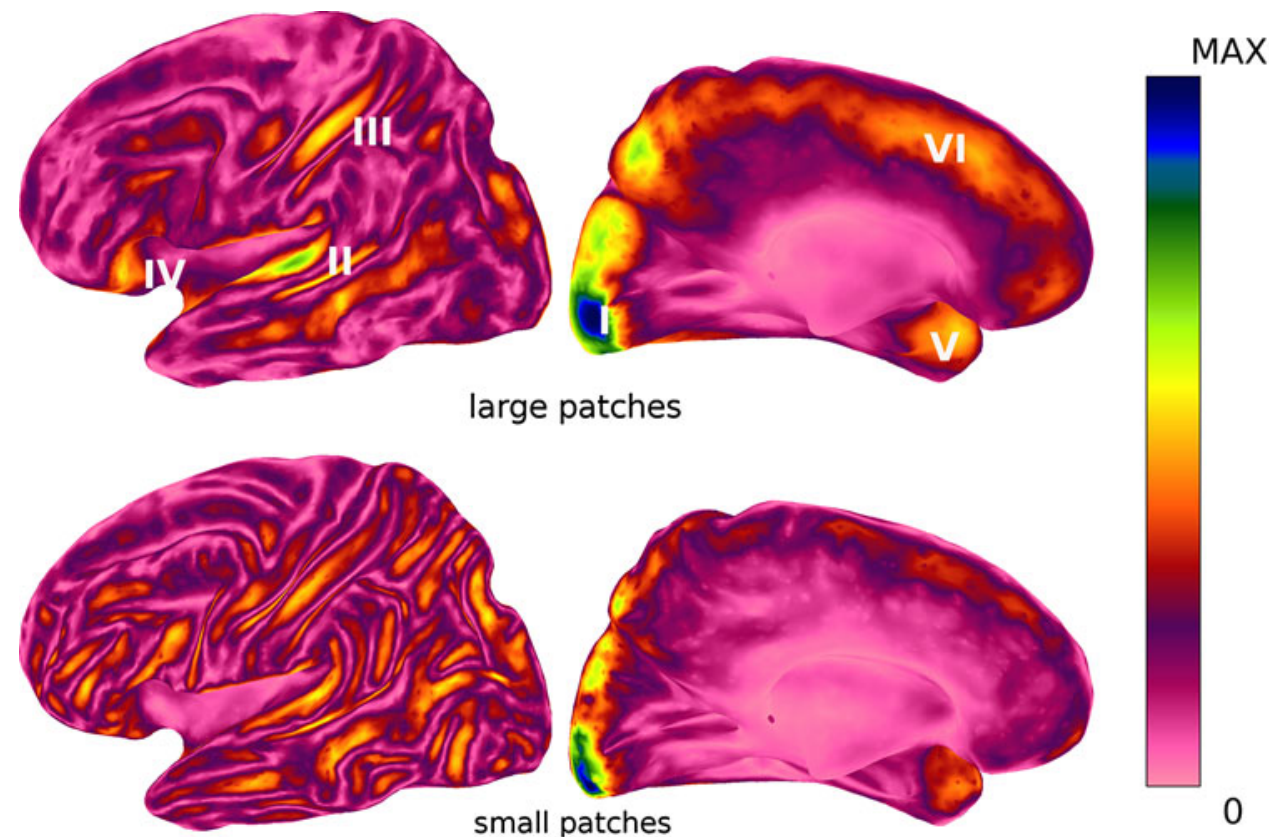

anatomical region. By ranking the various ECoG spikes according to a combined amplitude and synchronous surface area measure we found that MEG spikes could always be associated to larger, extended ECoG discharges. This suggests that, as is the case in EEG, a larger area needs to be synchronously active for a spike to be detected in MEG. Certainly not all ranked (only 56\%) ECoG spikes could be associated to a MEG result. This is especially the case in the mesial temporal region. In spite of the fact that many high amplitude spikes occur in this region for our patient population, few of them were seen in MEG (most that were seen showed fast propagation to sub-temporal regions). On the other hand, some areas stand out in the number of positive MEG associations (Table 1; Fig. 3). Although not all regions area equally covered in our patient group (e.g. for the occipital and parietal areas, where epilepsy surgery is often not considered, no significant conclusions can be drawn), especially for the fronto-orbital, inter-hemispheric, central and lateral temporal areas MEG seems a good indicator of interictal activity occurring in those regions.

It is tempting to hypothesize why this is the case. In the simulation study, we investigated the influence of local cortical orientation and curvature on the observed MEG amplitudes, by assuming that extended patches of cortex are synchronously active during an interictal spiking. We started out with the largest patch size $\left(\sim 300 \mathrm{~mm}^{2}\right)$ and dipole density $\left(200 \mathrm{pAm} / \mathrm{mm}^{2}\right)$ assumed for normal physiological activity in a similar study by Hillebrand and Barnes (2002), and compared results for even larger patch sizes $\left(\sim 1200 \mathrm{~mm}^{2}\right)$ assumed for epileptiform interictal spikes as suggested by the MEG-ECoG comparison. In Fig. 4 it is shown that there are a number of specific regions for which a high maximum MEG amplitude is generated, whereas in other regions it is much smaller. For the smaller patches this maximum amplitude is smaller but can occur more evenly distributed over the cortex. These specific regions for large patches correspond to areas found in the MEG-ECoG association for our large patient group (Fig. 1). They are typically regions where the cortical surface is relatively flat and tangentially oriented. Other regions, such as, e.g., the frontal medial gyrus, are curved and there large patches tend to contain substantial radial components whereas the tangential parts in the sulci partially cancel out. But even for large, tangential flat patches, deep areas such as the cingulate gyrus, insula or the mesial temporal lobe, that are often involved in epilepsy, remain undetectable for MEG. MEG will only selectively show the interictal spikes that can be detected in the intracranial ECoG, and this selection is not necessarily the one most relevant clinically.

The maximum MEG amplitudes found in the simulation study still appear to be relatively low $(\sim 1 \mathrm{pT})$. For rolandic childhood epilepsy, e.g., MEG spike amplitudes occurring around the post-central area can be as high as 5 pT (Huiskamp et al. 2004). This can be an indication that even larger patch areas have to be considered, or that abnormal synchrony associated with epilepsy can create dipole densities exceeding the value of $200 \mathrm{pAm} / \mathrm{mm}^{2}$ assumed in this study. These large focal spikes also often show spatio-temporal propagation. In future studies, amplitudes should be compared as well to simulations in which activation is propagated over large patches. In a recent study simulation results similar to the one presented here were presented (Goldenholz et al. 2009). Since these 
were based on a different anatomical MRI and triangulation, it is unlikely that results are typical for the geometry used in this study only.

Acknowledgements Pauly Ossenblok is acknowledged for providing part of the MEG data. Thom Oostendorp is thanked for supplying, and updating, software for volume conductor- and source modeling.

Open Access This article is distributed under the terms of the Creative Commons Attribution Noncommercial License which permits any noncommercial use, distribution, and reproduction in any medium, provided the original author(s) and source are credited.

\section{References}

Agirre-Arrizubieta Z, Huiskamp GJ, Ferrier CH, van Huffelen AC, Leijten FS (2009) Interictal magneto-encephalography and the irritative zone in the electrocorticogram. Brain 132(11):3060 3072

de Jongh A, de Munck JC, Gonçalves SI, Ossenblok P (2005) Differences in MEG/EEG epileptic spike yields explained by regional differences in signal-to-noise ratios. J Clin Neurophysiol 22(2):153-158
Goldenholz DM, Ahlfors SP, Hämäläinen MS, Sharon D, Ishitobi M, Vaina LM, Stufflebeam SM (2009) Mapping the signal-to-noiseratios of cortical sources in magnetoencephalography and electroencephalography. Hum Brain Mapp 30(4):1077-1086

Hillebrand A, Barnes GR (2002) A quantitative assessment of the sensitivity of whole-head MEG to activity in the adult human cortex. Neuroimage 16(3):638-650

Huiskamp GJ, van der Meij W, van Huffelen AC, van Nieuwenhuizen O (2004) High resolution spatio-temporal EEG-MEG analysis of rolandic spikes. J Clin Neurophysiol 21(2):84-95

Mosher JC, Lewis PS, Leahy RM (1992) Multiple dipole modeling and localization from spatio-temporal MEG data. IEEE Trans Biomed Eng 39(6):541-557

Rorden C, Brett M (2000) Stereotaxic display of brain lesions. Behav Neurol 12(4):191-200

Tao JX, Ray A, Hawes-Ebersole S, Ebersole JS (2005) Intracranial EEG substrates of scalp EEG interictal spikes. Epilepsia 46(5):669-676

van Oosterom A, Oostendorp TF, Huiskamp GJ, ter Brake HJ (1995) The magnetocardiogram as derived from electrocardiographic data. Circ Res 67(5):1503-1509

van t' Ent D, Manshanden I, Ossenblok P, Velis DN, de Munck JC, Verbunt JP, Lopes da Silva F (2003) Spike cluster analysis in neocortical localization related epilepsy yields clinically significant equivalent source localization results in MEG. Clin Neurophysiol 114(10):1948-1962 